\title{
Near-Death and Transcendental Experiences: Neurophysiological Correlates of Mystical Traditions
}

\author{
Jean-Pierre Jourdan, M.D. \\ Oraison, France
}

ABSTRACT: The first stages of transcendental experiences might be induced by blocking or saturating sensory input to the brain at the level of the hippocampus. This could be accomplished during near-death experiences by blockade of hippocampal NMDA receptors by endopsychosins and during meditation by alteration of the hippocampal theta rhythm. Mystical practices described in ancient yogic or taoist texts may also alter hippocampal theta by breath exercises or labyrinthine stimulation. Many accounts of kundalini awakenings are consistent with this theory, as is an extended version of Itzhak Bentov's physio-kundalini model.

Taking place at the approach of death, near-death experiences (NDEs) might be regarded as a good starting point for those who seek an answer to the question of survival. However, the same kind of NDE occurs in a physiological crisis-the classical NDE-as under psychological stress-the so-called "fear-death" experience (Stevenson, Cook, and McClean-Rice, 1989-90); and the same NDE can occur spontaneously or induced by various psychophysiological techniques in a mystical context. In fact, many aspects of the NDE and its long-term effects have been described both since time immemorial and in contemporary accounts, in most cases with no relationship to imminent death.

NDEs that occur in a true physiological crisis and those that occur under psychological stress can be compared in terms of the manner in

\footnotetext{
Jean-Pierre Jourdan, M.D., is a general practitioner in private practice and a founding member of the research committee of IANDS-France. Reprint requests should be addressed to Dr. Jourdan at 28 Av. Flourens Aillaud, 04700 Oraison, France.
} 
which they unfold, and in terms of their long-term effects. According to witnesses' reports, these long-term effects do occur, and can be disturbing; no serious near-death study can neglect them.

The question then arises: do we solve this problem of NDEs occurring without imminent death by deciding arbitrarily that all those who experience such phenomena, including important spiritual and religious figures, have suffered pathological hallucinations, or do we admit that those accounts are sufficiently similar and consistent to merit further study?

\section{The Phenomenology and Aftereffects of Near-Death Experiences}

As the unfolding of the NDE is by now quite well known, I will present here only some excerpts from French NDErs' accounts concerning essentially their impressions and aftereffects, showing how their concept of life, their sense of values, and their behavior are deeply modified after such experiences. A few examples may be more explicit than a long explanation.

One NDEr talked of "that divine part of man"; she had the impression that "in this life we only half live, and reality is after death," which she defined as a rebirth or awakening. Another said "in each human being exists a divine spark," and for her

earthly reality is an illusion because here on earth we cannot grasp the real sense of life.... I had the impression that my body was an integral part of the soil, the water, the sky, stars, and stones. I was both myself and everything at once; it's difficult to explain. ... I can't say that it was either joy or happiness; it was a sort of well-being that couldn't compare with beatitude, which I have never known. . . I saw nobody at the end of the tunnel; it was the infinite.

Concerning human beings, she said "they are tiny but allencompassing; it is for each individual to work towards the essence, but can words express this?"

Another spoke of the descent into infinite love, peace, and the feeling of infinite love reaching the absolute and the extraordinary and warming light in which she bathed.

These experiences are difficult to explain to those who have never known anything similar, due to our limited concepts and words, which seem pale and insufficient, somewhat like trying to translate a sym- 
phony into words and describe one's emotions listening to it. One experiencer, for example, admitted:

For a long time, I spoke to nobody of my experience for different reasons. First of all, I didn't want to use words to describe it because I felt they would diminish the splendor, because it was impossible to describe verbally. There was no point in talking about it, because anyway it would have been misunderstood.... It's very, very painful to keep it to myself.

During the NDE, there seems to be an opening into a different state of consciousness in which a vaster reality, transcending time, space, and matter encompasses ordinary reality. Apparently this opening does not disappear completely after the experience. It appears, as Kenneth Ring (1984) so aptly wrote, that a seed has been planted, free to germinate or not with time. A few quotations will illustrate this concept:

My sensitivity increased; sometimes I had premonitory dreams and often telepathic powers. I decide to call my mother or my daughter or else I think they will call me, and within five minutes I get a phone call. After this experience I left my body several times.

A more acute sensitivity, telepathic powers, and ability to leave one's body, as well as healing powers and the ability to help others.

Sensitivity is greater, I learn quicker, I can concentrate better, I have a better memory and psychic gifts, but most important, I strive to incarnate myself by trying to decrease out-of-body experiences. It is in and through the body that the experience of life is centered, and nowhere else.

By loosening the ties between consciousness and body, NDEs sometimes lead to many out-of-body experiences. In one instance, a man who became bedridden after a serious accident, in which he had a core NDE, experienced out-of-body experiences nearly every night. During these experiences, he had the sensation of complete identification with anything he fixed his attention on: by observing a tree, he became the tree, its leaves, its roots; he could feel the bird's nest in his branches. Another left his body at night during conscious sleep, an experience that enabled him to help dying people leave their bodies without fear.

These abilities are sometimes a burden, as it appears that precognitive phenomena often involve highly emotionally charged events. The future is not always bright, and even if, for NDErs, death is only the 
discarding of a worn-out body, some would willingly do without foreseeing the death of close friends and family:

I quickly discovered in the month that followed my experience that I knew how long people had left to live... Believe me, it's a very uncomfortable realization.

There is another, brighter, more common aspect: many discovered they had the power to help and heal others; altruism and compassion are leitmotifs in these testimonies. One NDEr said: "I develop more and more my capacity as a healing channel; divine energy uses me as its channel." The techniques used are varied, including laying on of hands, a sensation of acting upon the subtle body, shamanic techniques, nontraditional psychotherapy, and accompanying the dying.

Many NDErs describe synchronicities after their experience. Students of mystical tradition, either Oriental or Western, will recognize many characteristics of transcendental experiences and their aftereffects. Similar features are described in all mystical traditions, and occur spontaneously even outside those traditions: 25 percent of university students report having had at least one out-of-body experience (Palmer, 1979a), some of which are very similar to NDEs.

\section{Kundalini as a Model for Mystical Experience}

I will now focus on one tradition that has described particularly well this awakening to a higher consciousness and the techniques employed to reach it. Hindu tradition describes a "force" or "evolutionary power" called kundalini, symbolized by a snake coiled around the base of the spine. Its awakening and ascension up the spine open up six or seven "centers," called "chakras," along the body's axis. When kundalini reaches the last and highest chakra at the top of the skull, it awakens consciousness to a superior reality. Carl Jung was a forerunner in bringing this concept to Western scientists, having organized a seminar on kundalini in 1932. According to Lillian Silburn (1988, p. xiii):

Kundalini, the upright axis at the innermost center of a person and of the universe, is the very source of man's might, drawing and unfolding all his energies. However, rather than dwelling on the extraordinary powers acquired through her medium, the followers of the Trika, Krama, and Kaula systems lay stress on the peace and the living harmony she bestows. 
Still, this mysterious energy aroused by Kundalini yoga manifests with a violence beyond belief and cannot be manipulated without incurring certain risks.

Tara Michael wrote that

when kundalini sleeps in the Muladhara (the first and lowest chakra center), man awakes to the world. But when it awakes and unites with Shiva, man falls asleep to the world and becomes one with the infinite consciousness beyond matter. $(1979$, p. 34)

It is easy to compare this peace described by Silburn and the infinite consciousness described by Michael with sensations frequently reported in NDEs. Long-term changes that affect NDErs are precisely the changes found in yoga, and the Siddhis or powers that this awakening is supposed to confer-and in opposition to which exist many warnings-are precisely what many NDErs experience spontaneously: transcendence of time, in precognition; transcendence of space in telepathy, clairvoyance, and out-of-body experiences; and transcendence of matter in healing powers. Some experiences are physically and psychically disturbing.

The comparison between NDEs and the kundalini awakening concept has already been described by Ring (1984). One of the characteristics of kundalini is that it is accompanied by various physical, psychological, and sensory symptoms, which have been studied by Itzhak Bentov (1977), who advanced an explanation, Lee Sannella (1987), and Hiroshi Motoyama (Motoyama and Brown, 1978). Yoga has codified these characteristics, but it is worth noting that the same effects have been linked to a number of transcendental experiences around the world. It appears to be a universal phenomenon, at the origin of a set of experiences that humanity has always sought in order to transcend our condition. In order to provide a clearer idea of this kind of experience, I will briefly describe two of the better known examples, those of Gopi Krishna and Motoyama.

Krishna, a Hindu Brahman who had received a Western education, was in no way a mystic. His only tie with Hinduism was regular meditation. One morning, while meditating on a bright lotus on his head, he perceived a strange but pleasant sensation at the base of his spine, which fluctuated according to the intensity of his attention. When he concentrated again with determination, the sensation was renewed and it moved upwards: 
Suddenly, with a roar like that of a waterfall, I felt a stream of liquid light entering my brain through the spinal cord. ...

The illumination grew brighter and brighter, the roaring louder, I experienced a rocking sensation and then felt myself slipping out of my body, entirely enveloped in a halo of light.... I was now all consciousness, immersed in a sea of light simultaneously conscious and aware of every point . . . bathed in light and in a state of exaltation and happiness impossible to describe. (Krishna, 1971, pp. 12-13)

That was only the beginning of an experience that was to last years, with moments of exaltation alternating with periods of doubt and depression.

Motoyama, a Japanese scientist, related what he experienced over a period of several months after practicing the breathing techniques of pranayama yoga:

During continued practice, I began to notice some new sensations. I had an itchy feeling at the coccyx, a tingling feeling on the forehead and at the top of the head, and a feverish sensation in the lower abdomen. I could hear a sound something like the buzzing of bees around the coccyx. In ordinary daily life my sense of smell became so sensitive that I could not endure offensive odors.

These conditions continued for two or three months. One day, when I was meditating before the altar as usual, I felt particularly feverish in the lower abdomen and saw there a round blackish-red light like a ball of fire about to explode in the midst of a white vapor. Suddenly, an incredible power rushed through my spine to the top of the head and, though it lasted only a second or two, my body levitated off the floor a few centimeters. I was terrified. My whole body was burning, and a severe headache prevented me from doing anything all day. (Motoyama and Brown, 1978, pp. 240-241)

A few months later he started to have premonitory dreams and frequent telepathic experiences. His wishes seemed to be fulfilled spontaneously. Clairvoyance phenomena developed with the progressive awakening of the different chakras. Sometime after,

I saw a kind of heat energy rising from my coccyx to my heart through the spine.... As the kundalini rose from my heart to the top of my head, it became shining white. It left my body through the top of my head and I rose with it into a much higher dimension. (Motoyama and Brown, 1978, p. 247)

He then acquired a healing power. At the same time profound psychic changes took place, and he lost attachment to material things. The awakening of another chakra (the Vissudha chakra, situated at the 
throat level) gave him the ability to see the past, the present, and the future in the same dimension.

Similar concepts can be found in other traditions, implying the existence of a universal phenomenon. The Egyptian Book of the Dead (Budge, 1967) describes

a symbol of the vital fluid, of the fire snake in the spine. That fluid is the breath of life that the priest passes on by laying his hands on the deceased's nape of the neck to warm and cover him with Isis' heat.

For the Chinese Taoists (Brelet-Rueff, 1975),

Energy does not only flow in meridians; it also concentrates in certain areas linked by forces: the three "Fields of Cinnabar." The upper Cinnabar Field originates from the strange point Inn Trang (between the eyebrows). . . . The middle Cinnabar Field, or "Vermillion Palace," is situated on the chest with the heart as a center. ... It circulates energies. The lower Cinnabar Field-the third energy center... is two inches above the navel. The control of one's breath may allow the transmutation of mental energy into a golden elixir dripping the median canal to mix into ancestral energy and $\mathrm{Ki}$. In a burst of sparks, the substance created by the fusion of the $\mathrm{Ki}$, ancestral energy, and mental energy, flowing up through the central canal to the upper crucible, bursts the universal orbit of Tao into a gold, silver, sun and moon blossoming.

Anthropologist Richard Katz (1982) has studied the Kung, a tribe living in the Kalahari Desert. This tribe has a rite that seems to have a lot of common points with kundalini yoga: its aim is to awaken and set ablaze a force called num, in order to reach a state called kia, in which extraordinary possibilities appear, such as healing powers, remote viewing, and walking on fire. The num is said to lie in the pit of the stomach and the base of the spine. Once started, it flows up from the base of the spine to the skull, allowing people who reach the state of kia. One Kung healer described the action and ascent of num:

In your backbone you feel a pointed something, and it works its way up. The base of your spine is tingling, tingling, tingling, tingling. Then num makes your thoughts nothing in your head. (Katz, 1982, p. 42)

How is this phenomenon produced? Another Kung healer described the experience:

You dance, dance, dance, dance. Then num lifts you up in your belly and lifts you in your back, and you start to shiver. Num makes you tremble; it's hot. Your eyes are open, but you don't look around; you hold your eyes still and look straight ahead. But when you get into 
kia, you're looking around because you see everything, because you see what's troubling everybody. Rapid shallow breathing draws num up. ... Then num enters every part of your body, right to the tip of your feet and even your hair. (Katz, 1982, p. 42)

Sannella, a psychiatrist, is well known as a specialist in these phenomena. He has collected a number of similar contemporary testimonies in a book (1987). People who have gone through an NDE describe similar symptoms followed by similar consequences; an example is Barbara Harris's experience (Harris and Bascom, 1990; Ring, 1984).

Bentov (1977) and Sannella (1987) have collected the physiological symptoms occurring during kundalini awakening under the name of physio-kundalini. These symptoms can be grouped into three categories:

(a) sensory symptoms, including ticklings, vibrations or feelings of energy in a particular spot, flowing up the spine and then going down to the chest and abdomen, orgasmic sensations, pain starting and stopping suddenly, sensations of cold or heat in different parts of the body, various sounds, internal light sometimes illuminating the body; (b) motor symptoms, including spontaneous movements of hands and body, involuntary contractions of the anus, abdomen, or throat, changes in breathing rhythm, sudden locking or paralysis of certain parts of the body; and

(c) psychological symptoms, including sudden joy or ecstasy, anxiety or depression, acceleration of thought, and expansion of consciousness beyond the body.

\section{Neurobiological Mechanisms}

\section{The Role of Breathing}

One clue to what may happen in kundalini awakening is that most of the techniques used seem to aim at disconnecting consciousness of external stimuli from feelings, either by concentrating on a point, as in meditation on an object, a concept, a sound, or a posture; or by reciting a mantra or a prayer, or deep relaxation or contemplation. But one commonality that can be found nearly everywhere is the use of breathing, with, from the very start, the explicit notion of the equation:

$$
\text { mind }=\text { breath }
$$

Let me present a few examples. The first is a Taoist text, The Secret of the Golden Flower (Lao-tsu, 1991). To understand it, one must know two things: (a) that the same Chinese ideogram signifies both "heart" 
and "mind," and (b) that this ideogram is itself incorporated into the one that signifies "breathing."

Breathing comes from mind/heart. What comes out of that mind/heart is breathing. ... Since mind and breathing are interdependent, the revolution of light must be united to a rhythm given to breathing. ... The great saints, who were aware of how mind/heart and breathing energy influenced each other, made up a simplified method to help later generations. (p. 76)

In the Hatayoga Pradipika (II,2), "when breath is set into action, so is the spirit; when breathing stops, so does the mind" (Eliade, 1989b, p. 249). The goal of Tibetan yoga is to weaken this connection (EvansWentz, 1967, p. 139):

In consequence, that thing called mind-so difficult to control because of its normal incapacity to function outside the process of breathing which is the cause of the continuous impulses from one thought to another-takes control and gets free from that dependence on breathing. ...

The length of a thought is equal to that of a respiration.... These exercises aim at training the mind to work independently from breathing and therefore being no longer influenced by the process of thinking. Concepts grow up in the mind because of stimuli. Those stimuli must be neutralized and the process of thought disappears; then the mind reaches a natural state.

A similar process is described in the Vijnanabhairavatantra, "when one keeps in check the entire flow [of the sense activities] by means of the breath energy of breath which gradually rises" (Silburn, 1988, p. 66).

A praying technique, the Hesychasm, used by the monks of Mount Athos (Eliade, 1989b), consisted of controlling their breathing while repeating the words "Kyrie Eleison." And again, in the Kung tradition, "Rapid shallow breathing draws num up" (Katz, 1982, p. 42).

Numerous shamanistic techniques are based on breathing (Eliade, 1989a), as are the techniques used by famous mediums to facilitate their feats. Eileen Garrett, for instance, wrote:

In order to be clairvoyant it is possible for me by breath, and by breath alone, to lift myself above the normal everyday being. The moment I make contact with those who seek my help, I really, with animal understanding, "sniff" their atmosphere. Therefore, I think much of my clairvoyance could be a "leftover" primitive race faculty. I do exactly as the dog does when he catches the scent of a rabbit. I seize it 
and hold onto it until I know a good many needed facts about the personality of the inquirer. (1961, p. 61).

In each of these techniques, one must set a rhythm to one's breathing, either slowing it down, sometimes to the point of apnea, or speeding it up. The states reached by these techniques range from a more or less complete trance to a complete loss of contact with reality, producing a state that, according to tradition, might in its turn lead to a transcendent experience.

In order to sort out the main characteristics, I propose to start with, to put together all those phenomenologically similar experiences, without regard to the circumstances in which they take place. I call "nonordinary experience" any experience involving continued consciousness and memory and a feeling of reality, along with at least one of the following features:

1. a feeling of beatitude;

2. a feeling of leaving one's body;

3. transcendence of time and/or space;

4. perception by unusual channels, different from ordinary senses;

5. perception in an unusual form, such as panoramic vision, melting into or identification with an observed object or phenomenon, or nonverbal perception and comprehension;

6. corroborated access to information impossible to get by ordinary means;

7. perception of or participation in a transcendental event of a spiritual or mystical nature; or

8. long-term effects, such as restructuring, therapeutic, or evolutionary changes, or attainment of paranormal or healing abilities.

\section{Organization of Perceptions}

Visual, auditory, and tactile information detected by sensory organs is relayed through the thalamus before it reaches the cortex; that thalamic relay is responsible for orientation and focusing of attention on the sensory field corresponding to what is "new" in the environment. This information, focused by the thalamic relay, is then sent to the cortical area specific for each sensory organ, where it is analyzed and connected with corresponding information from the other hemi- 
sphere of the cortex. Once processed, that information is sent to the sensory association areas, where it is integrated and interpreted. Once there, these perceptions are modulated on the affective and emotional level by projections to the limbic cortex.

Olfactory information, by contrast, is processed differently from other sensory input, in that it is sent directly to the olfactory cortex without passing through the thalamus. Belonging anatomically to the limbic system, which appeared phylogenetically before the neocortex, the olfactory cortex has been driven to the center of the brain during evolution. The primary olfactory area is the pre-piriform cortex; the parahippocampal gyrus, or entorhinal cortex, is considered to be the secondary olfactory area.

At that point, we have a series of global but instantaneous representations of the external world. The instantaneous perceptions must still be linked together in time to be understood in a temporal context, allowing purely spatial perception to become spatiotemporal perception. The input from the associative areas is then sent, by way of the entorhinal cortex, to the hippocampus, where a memory bank accesses previous information and links it to the newest input. The information is linked temporally, allowing passage from the instantaneous to the present, and then sent back to the neocortex. This circuit with retroactive loops permanently reprocesses all sensory input. The processed information is then sent back to the neocortex, where semantic and cognitive integrations are made.

\section{Long-Term Potentiation, Hippocampal Theta, and Survival Behavior}

In order to explain learning and development in the brain, Donald Hebb (1949) propounded that neuronal activity should modify synaptic connections. That is, when one neuron stimulates another one often enough, metabolic or structural modifications of one or both cells should strengthen their connections. This hypothesis was unverifiable at that time, but in 1966, Terje Lomo discovered "long-term potentiation," a long-lasting enhancement of synaptic efficacy by which a neuron, by repeated neural input, is durably and sometimes permanently modified.

This phenomenon appears primarily in the hippocampus, at least in mammals, where memorization and learning seem to occur through long-term potentiation. A particular electrical activity called theta rhythm is at least one of the factors in this phenomenon, since theta 
seems to activate the N-methyl-D aspartate (NMDA) receptors that initiate long-term potentiation. That was proved by the fact that short trains of high frequency pulses, applied to certain parts of the hippocampus, induced long-term potentiation only if they were applied at the peak of theta rhythm, while they decreased synaptic efficacy if they were applied at the trough of theta activity.

Electrical recordings from the brains of rats in the waking state, in slow-wave sleep, and in rapid eye movement (REM) sleep have demonstrated that neuronal transmission in the hippocampus depends on behavior (Winson and Abzug, 1977). The hippocampal theta rhythm is linked to learned activity that is essential for survival, but not to genetically programmed behavior such as mating or feeding. For example, hippocampal theta appears in the cat as it hunts, in the rabbit when it senses a predator nearby, and in the rat as it explores its environment. In fact, the peaks of theta activity are synchronized with specific behaviors, such as sniffing the air and moving the whiskers or vibrissae.

This hippocampal theta rhythm seems to play a role in synchronizing the processing of sensory information; for instance, as the rat explores its environment, olfactory messages and tactile input from the vibrissae, together with the other sensory information, converge simultaneously on the hippocampus, where they are processed in 200millisecond bunches marked off by the theta rhythm. As Jonathan Winson wrote:

One may speculate that in animals such as the rat which make great use of the sense of smell it is important that all sensory information, touch sensations from the vibrissae, vision and hearing are coordinated with the cyclic inhalations of odors. In this way the entorhinal cortex, hippocampus and the rest of the limbic system can process all sensory input along with smell-an event is linked to its odor. (1985, p. 190)

I believe that this hippocampal theta rhythm may also function as an alarm signal for olfaction, the way the thalamic orientation reaction does for auditory, visual, and tactile perceptions. In those mammals in which olfactory messages provide information critical for survival, the theta rhythm would be a priority signal, indicating that information arriving at the time of peak theta activity should be considered vital and processed accordingly.

The hippocampal theta rhythm can also be evoked by rapid rotating motion, which stimulates the labyrinths of the inner ear (Costin, Bergmann, and Chaimovitz, 1967, p. 185): 
In the awake, non-stimulated animal, angular acceleration evoked a hippocampal theta-rhythm, expressing itself in synchronization and in an increase in amplitude.

The theta rhythm appears not only during survival-related behavior and as a result of labyrinthine stimulation, but also during REM sleep, even though no movement or information-seeking is occurring. Winson (1990) suggested that in REM sleep, the neocortex-hippocampus circuit, again alerted by the theta rhythm, could permanently reshape memories. Although theta activity during REM sleep is difficult to show in humans, he proposed that this phenomenon may be responsible at least in part for our dreams.

Long-term potentiation is produced by the activation of NMDA receptors in the granule cells of the hippocampus and in various areas in the neocortex. The neurotransmitter of these granule cells is the excitatory amino acid glutamate. While glutamate permits a quick transmission and processing of sensory influx, it can also lead to what is called excitotoxicity: if too much glutamate is produced, as can occur for example in anoxia, it becomes toxic and brings about the death of the neuron where it has been released.

\section{Biologically Produced Near-Death-Like Experiences}

Various experiences phenomenologically similar to NDEs can be produced by pharmaceutical agents or by physiological disturbances. Ketamine, a dissociative anesthetic, reduces the activity in the neocortex and subcortical structures, while it increases the activity in the limbic lobe and the reticular activating system. Its particular effect is to block NMDA receptors in the neocortex, thalamus, and hypothalamus. This disconnects the patient from sensory input, including pain, but does not really put him or her to sleep. Depending on the dose and the route of administration, it may leave consciousness and memory intact while progressively disconnecting sensory perceptions.

These effects seem to offer a reasonable experimental model for NDEs. In a study comparing the effects of anesthetics on consciousness, Barbara Collier (1972) gave 11 subjects 40 to 60 milligrams of ketamine intravenously, and had 11 other subjects inhale a mixture of 50 percent nitrous oxide and 50 percent oxygen. Of the 11 ketamine subjects, 10 reported a sensation of floating in space, nine felt the "spirit" or mind leave the body, four saw colored or white patterns with their eyes closed, three described "looking down" on their bodies on the gurney and noted the exact time their "spirit" re-entered it (which happened a few moments after the return of responsiveness to a light 
pinprick), two experienced the "mind" moving very rapidly through a vacuum with no sense of time or light, and one subject "became" one of a pile of boxes.

By contrast, of the 11 subjects who received the nitrous oxide, 10 experienced distorted body awareness, five experienced a sensation of floating upwards, four noted changes in auditory perception, two suffered from a sense of claustrophobia, and two became dizzy. Collier likened the descriptions given by the patients under the influence of ketamine to those given by subjects undergoing sensory deprivation.

Ketamine, at doses less than those used for anesthesia (generally between 0.4 and 0.6 milligrams per kilogram of body weight), has been used by psychiatrists to foster an emotional reliving of repressed stressful memories, leading to a therapeutic discharge of dammed-up emotions (Khorramzadeh and Lotfy, 1973). Six months after reviving memories with ketamine, 91 percent of patients with a variety of psychiatric disorders were considered cured.

Patients in that study reported experiences reminiscent of NDEs: "I was in a different world and with flash backs I was seeing vividly events which led to my illness," "I was talking to the Holy Family," "I was walking on an infinite piece of land and my life was marching in front of my eyes," "I was flying and chasing my own life," and "Heavy burden of $\sin$ is gone now" (Khorramzadeh and Lotfy, 1973, p. 345).

Another psychiatrist, Ladislas J. von Meduna (1950), tried treating various neuropsychiatric disorders with a mixture of 70 percent oxygen and 30 percent carbon dioxide. This mixture induced experiences that included certain elements of an NDE, such as a feeling of getting out of one's body, a vision of a bright light, and ineffability. However, the experience was accompanied by severe neurological disturbances and unpleasant hallucinations.

Experimental stimulation of the temporal lobes also seems to produce comparable phenomena. Wilder Penfield $(1955,1975)$ described the results of experiments in electrical stimulation of various zones of the temporal lobes during surgery for temporal lobe epilepsy. The patients, who had not been put to sleep, could describe their perceptions, and when Penfield stimulated various regions of the temporal lobes, they reported such phenomena as motor responses, sensory or somatic illusions, feelings of vertigo, and of leaving one's body, as well as more complex phenomena such as sensations of deja $v u$, hearing pieces of music, remembering whole blocks of memories, and a sense of reliving certain memories.

For example, Penfield reported the following patient's responses to electrical stimulation of a particular point: 
Stimulation at a depth of $2 \mathrm{~cm}$. along the superior surface of the temporal lobe, within the fissure of Sylvius, caused the patient to say suddenly, "That bitter-sweet taste on my tongue." He seemed confused and made tasting and swallowing movements.

The stimulating current was shut off and the electro-corticogram showed that a slow wave 4 per second generalized rhythm had been set up as an after-discharge. While this was continuing the patient exclaimed: "Oh God! I am leaving my body." Dr. Karagulla, who was observing him, said he looked terrified at the time of the exclamation and made gestures as though he sought help. When the electrocorticogram had returned to normal the patient seemed to be himself again. (Penfield, 1955, p. 458)

Penfield hypothesized on the basis of such observations that superficial stimulation of the temporal lobe can in fact act on a deeper zone with which it is directly linked and which is, among other things, in charge of storing memories. He speculated that the hippocampus might be that deeper zone, and since then strong monosynaptic connections have been discovered between the temporal cortex and the limbic structures, particularly with the hippocampus.

Karl Jansen (1990) proposed a hypothesis based on the fact that ketamine acts by blocking NMDA receptors, especially in the hippocampus, preventing long-term potentiation. That blockade would amount to closing the access channel to sensory information, whereupon, Jansen proposed, internal memories would take the place of the missing sensory input.

The discovery of endogenous ligands for those very receptors supported Jansen's hypothesis: those ligands, called endopsychosins, could be released in anoxia in order to block access of glutamate to the NMDA receptors and thus prevent the death of the target neuron by excitotoxicity (Contreras, Monahan, Lanthorn, Pullan, DiMaggio, Handelmann, Gray, and O'Donohue, 1987). The result would then be similar to what occurs with ketamine and could explain the phenomenon of the life review or panoramic memory found in many NDEs.

\section{Sensory Disconnection and Spiritual Experiences}

Before proceeding, let me clarify that this model attempts to address the spiritual, which is beyond words and beyond common experience. It is virtually impossible to compare, let alone try to explain, intimate experiences that are well beyond the human capacity for analysis and understanding. All we can do for the moment is to try to catch a 
tangible thread common to these experiences, and then pull on it to see what will come next. This thread may reside in the various factors that are at the origin of the experience. If we could isolate a common neuropsychophysiological substrate, we might then be able to carry out objective research based upon more than just speculation and phenomenology.

As noted above, Winson (1990) proposed that REM sleep is linked to a reprocessing of the information received while awake and to a reshaping of memory by induction of a hippocampal theta rhythm. That rhythm, which under certain circumstances is closely yoked to the breathing pattern in frequency and phase, seems to be able to modulate information processing during wakefulness as well as during sleep. Similar effects can be produced by pharmacological agents such as ketamine, or by other means, such as in hypercapnia or electrical stimulation. That fact might explain the cathartic effect seen with ketamine as well as in the modified states of consciousness induced by using various breathing techniques or after an NDE.

Furthermore, if we assume that memory reorganization during REM sleep induces a theta rhythm in the neocortical-hippocampal circuit, then inducing an artificial theta rhythm by changing the breathing pattern or by body movements that stimulate the labyrinth of the inner ear could produce the same result. If these techniques also produce sensory and motor disconnection, another characteristic of REM sleep, then every condition that seems to be at the base of a number of nonordinary experiences is present.

The hippocampus is an archaic structure of the brain that used to play a critical role in the sense of smell. While its role in olfaction has since passed on to the entorhinal area, which also receives and processes interoceptive information, the circuits that modulate functioning of the hippocampus may still exist in humans. As we have already noted, the influx from the sensory cortex reaches the hippocampus through the entorhinal cortex, which, since it is still directly linked to the sense of smell, might be modulated by a particular breathing rhythm. If we assume that the theta rhythm could in fact be, for olfaction, the equivalent of the thalamic orientation reaction for the other senses, then changing one's breathing rhythm intentionally might deceive certain circuits and give priority to olfactory and interoceptive information, inhibiting the transmission of other sensory information (Motoyama and Brown, 1978).

But we are not a particularly gifted species when it comes to olfaction, and if there is furthermore no particular smell to perceive, then limiting our attention to olfactory input could in many cases focus our 
consciousness on nothing at all, producing a sensory disconnection. Most of the techniques used in mystical traditions involve mastering the breathing pattern or, as in the shaman's dancing and the dervish's spinning, stimulating the labyrinth, which may produce bursts of hippocampal theta rhythm. In fact, it is likely that any voluntary change in the breathing rhythm will have some effect on the processing of information by the brain.

Apart from sensory deprivation experiments, in which there are virtually no environmental data to be processed, all the mechanisms mentioned above deal with data processing in the hippocampus and its related structures. A hypothesis can be deduced from all this that NDEs are part of a large number of experiences, which I call nonordinary experiences, that all have as the same starting point a sensory disconnection. Since there is an emotional detachment, particularly in the NDE, there seems to be a disconnection of certain parts of the limbic system as well, which corroborates this hypothesis.

That disconnection, during the NDE, might be due to the release of neuroprotective substances blocking the NMDA receptors; Jansen (1990) proposed this mechanism as an explanation for the life review. Voluntary induction of similar experiences by breathing exercises could be mediated by a progressive blockade of hippocampal transmission, allowed by the persistence of archaic ways of modulating perceptions at that level.

That hippocampal blockade may also occur in the "fear-death experiences" (Stevenson, Cook, and McClean-Rice, 1989-90), which may be related to "stress-induced antinociception," in which disturbance of serotonergic systems reduce the response to pain stimuli under stress (Carr, 1982; Morse, Venecia, and Milstein, 1989; Snow, Tucker, and Dewey, 1982). Since afferent pathways of the hippocampus are serotonergic, one of the bases of Melvin Morse, David Venecia, and Jerrold Milstein's (1989) hypothesis on NDEs, the analgesia in stressinduced antinociception may be produced by a modulation in the hippocampus. That modulation could sometimes be enough to start a complete disconnection, thus creating the conditions allowing an NDEequivalent to happen.

In the experiments on hypercapnia, the increase in carbon dioxide could be thought of as the beginning of anoxia, which would release neuroprotective substances. The high levels of carbon dioxide would be responsible for side effects producing distortion and the unpleasant aspect of the perceptions, perhaps through a hyperexcitability of the temporal lobe. In the experiments using ketamine, action roughly similar to that of the endopsychosins induces at the least a flux of 
memories, and with a larger dose a more complete disconnection, precipitating an out-of-body experience.

In the case of the Penfield experiments quoted above, since the sensation induced by the stimulation of the temporal lobe was gustatory, the piriform lobe of the entorhinal cortex may have been indirectly stimulated. The out-of-body experience described happened after the stimulation had stopped, while a synchronized rhythm was in process; that synchronization might have disrupted the hippocampus by imitating a theta rhythm, or the recorded rhythm may have itself been a hippocampal afterdischarge having diffused to the temporal lobe.

As for the experiences reported during pure meditation, the link between hippocampal and cortical theta rhythm is not as clear. However, the EEG recordings of experienced yogis during meditation showed slow rhythms, first alpha and then theta becoming more prominent (Twemlow, Gabbard, and Jones, 1982). A study by John Palmer (1979b) showed that out of 20 subjects, the three who had the most lively out-of-body experiences had more than 30 percent theta waves in their EEG, implying at least a certain disconnection from their environment.

\section{The Role of the Hippocampus in Kundalini}

It is therefore possible to describe a neurophysiological mechanism for the initiation of non-ordinary experiences, a mechanism concerning the hippocampus as the essential key formation. Can we go further? The hypothesis I propose, in which the functioning of the hippocampus is altered by external factors, seems if not to explain at least to allow an interpretation of some of the collateral phenomena described in mystical traditions and confirmed by contemporary experiences.

Every account of these non-ordinary experiences includes a host of various sensations described above as comprising the physio-kundalini syndrome. Although those phenomena occur in a mystical context, they do have a reality nevertheless for those who experience them. The question is then: What do they reflect? To answer this question, let us review a few premises.

The kundalini awakening is said to allow an evolution from or a transcendence above the ordinary human condition. Kundalini awakening must then be accompanied by actual changes, both physiological and psychological.

Our nervous system is malleable enough so that the way we function is not fixed rigidly by strict laws, but can be altered either unconsciously by adaptive mechanisms or more or less willingly through 
certain techniques. Some techniques such as biofeedback are known to be capable of causing lasting modifications in vegetative functions that are normally not under voluntary control (Green and Green, 1977).

If the sporadically occurring physio-kundalini phenomena affect all the cerebral systems, then at the peak of these experiences there is what seems to be an actual explosion, a saturation of all transmitting channels into synesthesia. This is illustrated by Krishna's description of his kundalini awakening, cited above (1971, pp. 12-13):

Suddenly, with a roar like that of a waterfall [auditory], I felt [somaesthetic] a stream of liquid [tactile] light [visual] entering my brain through the spinal cord.... I felt a rocking sensation [equilibrium] and then felt myself slipping out of my body. ... (Krishna, 1971, pp. 12-13)

This seems to be a general excitation, perhaps such an unusual phenomenon that each sense tries to express in its own way.

A superficial survey of these perceptions can of course lead to the impression that they are pathological brain disturbances. Some people presenting with those symptoms have rather quickly been labeled and treated as psychotics. Krishna (1971) and Sannella (1987) both estimated that about 30 percent of patients labeled as psychotic or schizophrenic are in fact victims of a pathological "awakening." Krishna gave as examples the characters called Avahoots in India and Mastanas in Persia, who combine extraordinary gifts of clairvoyance with schizophrenia and manic-depressive psychosis at different stages.

This is not the proper place to discuss the relationship between madness and transcendence. However, it is remarkable that psychosis is manifested particularly by identity problems and by the loss of reality testing, which are not found in kundalini experiences. And while some physio-kundalini symptoms resemble epilepsy in their location and progression, seizures are always associated with cerebral irritation, whether that is due to trauma, tumor, or vascular lesion. When the seizure is generalized, it is a short dramatic event leading to a loss of consciousness with subsequent amnesia; no such pathology is found in kundalini phenomena.

Every mystical tradition attempts to integrate the lower levels of the psyche by controlling the behaviors, instincts, feelings, and perceptions of the mind. This control may be functional as well as psychological; that is, the working of the nervous system may be altered, since the deepest and most archaic structures, the limbic system and reptilian brain, must be mastered and integrated by higher structures. This seems to be the case with the long-term aftereffects found in 
NDErs and in transcendental experiencers generally: altruism and empathy, which are the most important aftereffects, first appear phylogenetically with the development of the prefrontal cortex (McLean, 1990).

The hippocampus is a central processing area at which meet the sensory and associative cortex, which are turned outwards; the limbic system, which is turned both inwards and outwards and helps perceptions acquire an emotional value; memory; and the prefrontal cortex, which makes us human. Any phenomenon dealing with hippocampal functioning is therefore liable to modify all those areas.

Many of the facts reviewed above point to changes in hippocampal functioning. First, the hippocampus is particularly rich in NMDA receptors, which can also be found in the thalamus and neocortex. Second, those receptors are now known to be one of the main factors in the synaptic regression that is at the heart of cerebral development and plasticity. Synaptic regression is not, as its name might seem to imply, a degenerative phenomenon; as in creating a sculpture, the more detail you want, the more substance you have to take away.

Third, NMDA receptors are also the site of long-term potentiation, which may alter, sometimes forever, neuronal status. Fourth, longterm potentiation can be influenced, again through NMDA receptors, by both endogenous and exogenous factors, and probably by voluntary changes in breathing rhythm. Finally, whereas most parts of the brain have only brief periods of plasticity, generally during their embryonic development, the hippocampus retains this capacity throughout life.

These facts allow a few speculations. In the yogic tradition, the techniques of meditation and visualization concern all the senses; one can meditate or concentrate on a sound, a posture, a symbol, a concept, on various parts of the body, particularly by visualizing the various chakras at their own level, or on the circulation of energy or prana, which is said to be the subtle component of breath. Meditation is used together with techniques controlling the breathing rhythm, which are of prime importance in every tradition.

As noted above, interoceptive and olfactory information go directly to the entorhinal cortex; I propose that the theta rhythm may serve as a priority signal for them as well under certain circumstances. If it is true that certain breathing techniques can modify the processing of sensory information in the hippocampus, then it is possible that exteroceptive information could be inhibited while interoceptive information, traveling through another channel, could be treated as a priority transmission. 
An enhanced perception of normally unconscious inner phenomena is a necessary and sufficient condition to create a feedback loop that would in turn allow one to increase or decrease these inner sensations. Physio-kundalini could well be the consequence of an increased perception of both (a) normally unconscious interoceptive information and (b) the functioning of certain parts of the nervous system, which would be translated differently by each sensory system.

The various techniques for concentrating and visualizing could start a feedback loop, initiated and reinforced by breathing techniques, in turn creating durable modifications in the functioning of certain parts of the nervous system through the phenomena responsible for neuronal plasticity, long-term potentiation and synaptic regression.

Since the hippocampus has centrifugal connections with the hypothalamus and other structures dealing with somatovisceral, emotional, and endocrine functions, we can then understand the possibility of actual changes in all those functions, which, according to some reports, are not always positive nor well tolerated. Indeed, this mechanism may provide an elegant explanation for what is called mystic physiology, involving the chakras with their various functions and the "nadis," through which flow energy or prana. According to this hypothesis, this mystic physiology is more like a projection or corporal interpretation of neuronal functioning than any anatomic reality.

The fact that in some animal species the hippocampal theta rhythm is linked to breathing frequency is not of course evidence that the same is true in humans. However, given that most mystical traditions use rhythm and breathing changes to induce altered states of consciousness, the persistence of an archaic circuit allowing the modulation of perceptions and states of consciousness in humans cannot be ruled out.

The hippocampus is a crossroads between perceptions, memory, and consciousness. If all the phenomena described above are capable, in one way or another, of influencing hippocampal functioning, then they can also modify the relationship of consciousness with the outside world. That world is usually perceived through the sensory organs, which are filters as well as sensors, and they give us but a hazy image of the outside world. In fact the world we know is not an objective reality, but rather only a representation of one, albeit consensual.

The hypotheses I propose concern certain characteristics of brain function that could allow a non-ordinary experience and could possibly help us understand some of their long-term effects; but I do not claim that they explain the experience itself. Unusual perceptions, difficulty telling others about one's experience, and deep changes in one's con- 
cept of space and time during non-ordinary experiences suggest that they are perceived without the usual cortical tools of perception and cognition. In numerous cases, the acquisition of information supposedly unobtainable rules out any hypothesis that these experiences are hallucinations or purely neurological phenomena.

Concerning NDEs, the same experience may occur either in an unharmed brain or in an anesthetized person undergoing cardiac arrest, subject to a combination of anoxia, hypercapnia, anesthetic chemicals, and metabolic intoxication. This leads to the question of how this experience can occur and be processed into memory in the same way under such different brain conditions.

The deeply spiritual aspect of these experiences eludes for the moment any objective research, but it exists nonetheless. I hope I have shown that it is possible to draw a bridge, however frail, between science and transcendence, and that spiritual matters can be studied without necessarily making the opposing mistakes of either a scientific reductionism or a blind mysticism, which may be but differing appearances of the same lack of curiosity.

\section{References}

Bentov, I. (1977). Stalking the wild pendulum: On the mechanics of consciousness. New York, NY: Dutton.

Brelet-Rueff, C. (1975). Les médecines sacrées. Paris, France: C.R.L.T.

Budge, E.A.W. (1967). The Egyptian book of the dead: The papyrus of Ani in the British Museum. New York, NY: Dover.

Carr, D.B. (1982). Pathophysiology of stress-induced limbic lobe dysfunction: A hypothesis for NDEs. Anabiosis: The Journal of Near-Death Studies, 2, 75-89.

Collier, B. (1972). Ketamine and the conscious mind. Anaesthesia, 27, 120-134.

Contreras, P.C., Monahan, J.B., Lanthorn, T.H., Pullan, L.M., DiMaggio, D.A., Handelmann, D.E., Gray, N.M., and O'Donohue, T.L. (1987). Phencyclidine: Physiological actions, interactions with excitatory amino acids and endogenous ligands. Molecular Neurobiology, 1, 191-211.

Costin, A., Bergmann, F., and Chaimovitz, M. (1967). Influence of labyrinthine stimulation on hippocampal activity. In W.R. Adey and T. Tokizane (Eds.), Structure and function of the limbic system. Progress in brain research, vol 27 (pp. 183-188). Amsterdam, The Netherlands: Elsevier.

Eliade, M. (1989a). Shamanism: Archaic techniques of ecstasy. New York, NY: Viking Penguin.

Eliade, M. (1989b). Yoga: Immortality and freedom New York, NY: Viking Penguin.

Evans-Wentz, W.Y. (1967). Tibetan yoga and secret doctrines. New York, NY: Oxford University Press.

Garrett, E. (1961). Psychopharmacological parallels to mediumship. New York, NY: Parapsychology Foundation.

Green, E., and Green, A. (1977). Beyond biofeedback. New York, NY: Delacorte.

Harris, B., and Bascom, L.C. (1990). Full circle: The near-death experience and beyond. New York, NY: Pocket Books. 
Hebb, D.O. (1949). The organization of behavior: A neuropsychological theory. New York, NY: Wiley.

Jansen, K.L.R. (1990). Neuroscience and the near-death experience: Roles for the NMDA-PCP receptor, the sigma receptor and the endopsychosins. Medical Hypotheses, $31,25-29$.

Katz, R. (1982). Boiling energy: Community healing among the Kalahari Kung. Cambridge, MA: Harvard University Press.

Korramzadeh, E., and Lotfy, A.O. (1973). The use of ketamine in psychiatry. Psychosomatics, 14, 344-346.

Krishna, G. (1971). Kundalini: The evolutionary energy in man. Boulder, CO: Shambhala.

Lao-tzu. (1991). The secret of the golden flower. (Trans. by T. Cleary). San Francisco, CA: Harper.

Lomo, T. (1966). Frequency potentiation of excitatory synaptic activity in the dentate area of the hippocampal formation. Acta Physiologica Scandinavica, 68, Suppl. 277, 128.

McLean, P.D. (1990). The triune brain in evolution: Role in paleocerebral functions. New York, NY: Plenum.

Meduna, L.J. von. (1950). Carbon dioxide therapy: A neurophysiological treatment of nervous disorders. Springfield, IL: Charles C. Thomas.

Mesulam, M.-M. (1981). Dissociative states with abnormal temporal lobe EEG: Multiple personality and the illusion of possession. Archives of Neurology, 38, 176-181.

Michael, T. (1979). Corps subtil et corps causal Paris, France: Courrier du Livre.

Morse, M.L. Venecia, D., Jr., and Milstein, J. (1989). Near-death experiences: A neurophysiological explanatory model. Journal of Near-Death Studies, 8, 45-53.

Motoyama, H., and Brown, R. (1978). Science and the evolution of consciousness: Chakras, ki, and psi. Brookline, MA: Autumn Press.

Palmer, J. (1979a). A community mail survey of psychic experiences. Journal of the American Society for Psychical Research, 73, 221-251.

Palmer, J. (1979b). ESP and out-of-body experiences: EEG correlates. In Roll, W. (Ed.), Research in parapsychology, 1978: Abstracts and papers from the 21 st annual convention of the Parapsychological Association (pp. 135-138). Metuchen, NJ: Scarecrow Press.

Pavlides, C., Greenstein, Y., Grudman, M., and Winson, J. (1988). Long-term potentiation in the dentate gyrus is induced preferentially on the positive phase of thetarhythm. Brain Research, 439, 383-387.

Penfield, W. (1955). The role of the temporal cortex in certain psychical phenomena. Journal of Mental Science, 101, 451-465.

Penfield, W. (1975). The mystery of the mind. Princeton, NJ: Princeton University Press.

Ring, K. (1984). Heading toward omega: In search of the meaning of the near-death experience. New York, NY: Morrow.

Sannella, L. (1987). The kundalini experience: Psychosis or transcendence? Lower Lake, CA: Integral Publishing.

Silburn, L. (1988). Kundalini: The energy of the depths. Albany, NY: State University of New York Press.

Snow, A.E., Tucker, S.M., and Dewey, W.L. (1982). The role of neurotransmitters in stress-induced antinociception (SLA). Pharmacology, Biochemistry and Behavior, 16, 47-50.

Stevenson, I., Cook, E.W., and McClean-Rice, N. (1989-90). Are persons reporting "neardeath experiences" really near death? A study of medical records. Omega, 20, 45-54.

Twemlow, S.W., Gabbard, G.O., and Jones, F.C. (1982). The out-of-body experience: A phenomenological typology based on questionnaire responses. American Journal of Psychiatry, 139, 450-455.

Winson, J. (1985). Brain and psyche: The biology of the unconscious. Garden City, NY: Doubleday.

Winson, J. (1990). The meaning of dreams. Scientific American, 263(5), 86-96. 
Winson, J., and Abzug, C. (1977). Gating of neuronal transmission in the hippocampus: Efficacy of transmission varies with behavioral state. Science, 196, 1223-1225. 